\title{
SYNTHETIC SEX RATIO DISTORTERS BASED ON CRISPR FOR THE CONTROL OF HARMFUL INSECT POPULATIONS
}

\author{
B. FASULO ${ }^{1}$, A. MECCARIELLO ${ }^{1}$, P. A. PAPATHANOS ${ }^{2}$ AND \\ N. WINDBICHLER ${ }^{1}$
}

\author{
${ }^{1}$ Imperial College London, Department of Life Sciences, Imperial College Road, \\ London,SW7 2AZ,UK; nikolai.windbichler@imperial.ac.uk \\ ${ }^{2}$ Department of Experimental Medicine, University of Perugia, Perugia, Italy
}

\begin{abstract}
SUMMARY
Since the overall reproductive output of a population is typically determined by the fertility of its females, which are rate-limiting in gamete production, a successful way to genetically control a population should involve artificially biasing the sex ratio towards males. In male heterogametic species, this could be achieved by the expression of a transgene-encoded endonuclease during spermatogenesis that would target and "shred" the X chromosome at several loci. This would prevent the transmission of X chromosome bearing gametes to the progeny, generating only males. Recent developments in molecular and synthetic biology have provided genome editing tools with great potential to engineer the genome of different species. Given the targeting flexibility of CRISPR-based endonucleases, it may now be possible to test whether X chromosome shredding has the potential to become a universal strategy to genetically control a wide variety of insect pests, of both agricultural and public health relevance.
\end{abstract}

Key Words: Meiotic drive, sex ratio distorters, biased sex ratio, gene editing, genome editing, X-shredding, genetic control, spermatogenesis, male-determining genes, gene drive, selfish chromosomes

\section{INTRODUCTION}

In most sexually reproducing organisms, males and females are generated in approximately the same numbers. Fisher's principle states that the sex ratio is in equilibrium when an individual spends the same amount of energy to produce equal numbers of males and females. When the ratio is different from the equilibrium, the less predominant sex, or rather genes determining development towards this sex, will have an advantage that will last until the equilibrium is re-established (Fisher 1958). As the balanced sex ratio is approached, the advantage associated with producing the

J. Hendrichs, R. Pereira and M. J. B. Vreysen (eds.), Area-Wide Integrated Pest Management: Development and Field Application, pp. 843-855. CRC Press, Boca Raton, Florida, USA. (C) 2021 IAEA 
rarer sex wanes, and the equilibrium is re-established. Consequently, novel genetic traits that bias the sex ratio towards one sex gain a short-term advantage but are eventually counterbalanced by a neutralizing evolutionary force in the form of drive suppressors that evolve on the autosomes or on the Y chromosome. Sex ratios can therefore be portrayed as the dynamic outcome of an ongoing evolutionary "tug of war" (Argasinski 2013). Fisher's principle only applies in those cases where the sexratio is controlled by genes acting in the homogametic sex, or by autosomal genes acting in the heterogametic sex, in XX-XY and ZW-ZZ systems.

In a population of a sexually reproducing organism, a dramatic sex bias towards one of the two sexes usually decreases the population's overall fertility. Since the overall reproductive output of a population is typically determined by the fertility of its females, which are rate-limiting in gamete production, a successful way to genetically control the population could involve artificially biasing the sex ratio towards males (Hamilton 1967). Such a genetic control strategy could reduce the size of harmful animal populations, such as agricultural insect pests or disease-vector species, or even result in the suppression or collapse of the population before suppressor alleles can arise to re-establish a balanced sex ratio. In control programmes, a male-biased sex ratio would also be favourable because females are often responsible for the damage (e.g. they are often the vectors of human parasites or viruses (e.g. mosquitoes) or lay eggs in agricultural products (e.g. fruit flies).

Hamilton was among the first to suggest how genetic sex ratio distorters (SRDs) could be applied to eradicate mosquito populations, and that under certain conditions, non-Fisherian sex ratios could arise and yet be maintained. He considered a heterogametic species with males (XY) harbouring a mutant $\mathrm{Y}$ chromosome that can bias fertilization in its favour at the cost of the $X$ chromosome. Males carrying such a Y mutation would only produce sons. As a result, the mutant Y chromosome would gain a selective advantage and spread within the population, rendering it increasingly male-biased at each generation. The decline in female numbers would result in a decrease in size and eventually the collapse of the population (Hamilton 1967). In this example of SRD, an invasive $\mathrm{Y}$ chromosome interferes with the production of $\mathrm{X}$ bearing gametes during spermatogenesis and spreads through the population in a selfsustaining manner. Hamilton's thought experiment was inspired by observations of natural populations of mosquitoes, where Craig et al. (1960) reported that a SRD transmitted by males was responsible for a male bias in Aedes aegypti (L.), the yellow fever mosquito. Observations in other species since then have also identified an $\mathrm{X}$ chromosome bias and thus more female progeny in some populations of Drosophila simulans Sturtevant (Mercot et al. 1995). Importantly, it is often difficult to identify the existence of a SRD in a population because local suppressors evolved to counteract it - had they not the population would have likely disappeared.

For the purpose of this chapter, it is important to distinguish such driving (invasive) SRDs from traits that bias the sex ratio, but that are inherited in a nondriving standard Mendelian fashion (Fig. 1). Both types of SRDs, in turn, are distinct from female killing systems (FK) that also result in a biased sex ratio in progeny, but they do so at the expense of reproductive output because of post-zygotically lethality of female offspring. 
Unlike the bias generated by female killing systems (FK), SRDs operate prezygotically during gametogenesis and thus do not result in an overall reduction of male fertility. The main advantage of driving SRDs, as far as genetic population control is concerned, is their invasiveness. In the absence of resistance against the drive, the driving $\mathrm{Y}$ chromosome will eliminate the $\mathrm{X}$ chromosome in sperm and eventually lead to population collapse due to the lack of females.

Nevertheless, non-invasive SRD traits also have significant potential for genetic control, although they are eventually lost in the absence of continuous releases. They are advantageous in particular when compared to other forms of inundative genetic control approaches such as the Sterile Insect Technique (SIT) (Dyck et al. 2021) or its transgenic cousin, the Release of Insects carrying a Dominant Lethal (RIDL technology) (Thomas et al. 2000) as they require smaller effective releases (Schliekelman et al. 2005).

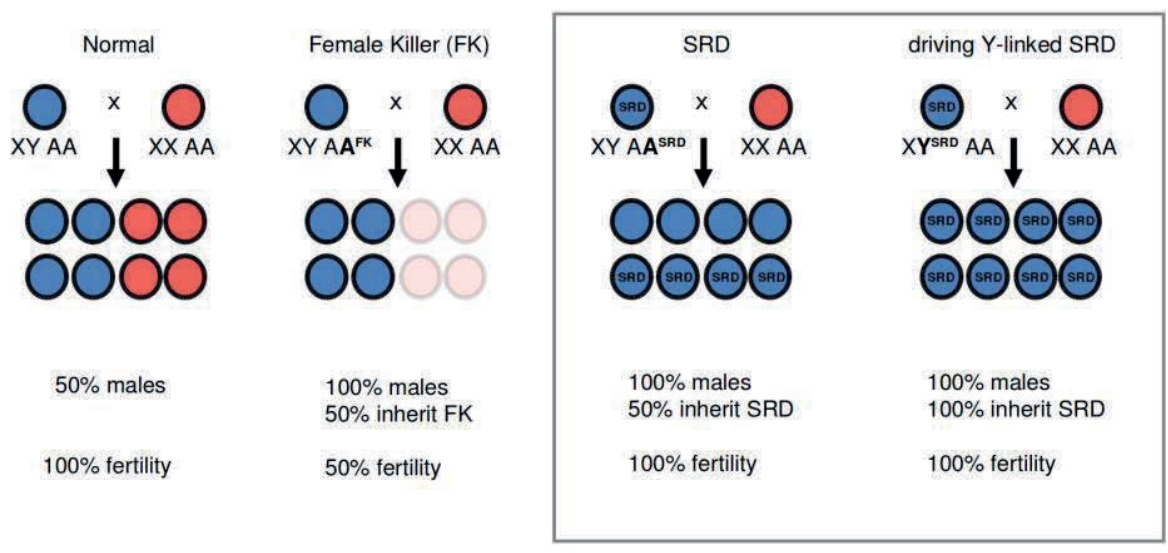

Figure 1. Male-biased sex ratio distorters (SRDs), unlike female killing systems (FK), bias the reproductive sex ratio towards males, while ideally maintaining full fertility.

$\mathrm{SRD}$ is a form of segregation distortion (SD) or meiotic drive, a term that also encompasses transmission of anomalies that are not strictly meiotic but that alter the normal process of meiosis, generating a gametic pool with one type of allele in excess (Zimmering et al. 1970). When SDs are physically linked to sex-determining loci or sex chromosomes, meiotic drive will result in an unbalanced sex ratio in the next generation. Reduced recombination between sex chromosomes favours the emergence of meiotic drive systems along them and indeed sex chromosome SDs are abundant in nature (Hammer 1991; Lyttle 1991).

SRDs occur mostly in association with male heterogamy, and usually, it is the $\mathrm{X}$ chromosome that drives against the $\mathrm{Y}$ chromosome, as a consequence of which males produce a strongly female-biased progeny. However, SRD systems in which the X drives against the Y chromosome, as in Drosophila, are not efficient in insect control programmes. Not only does population collapse proceed at a slower pace and is delayed by male polygamy, but it could result in short-term spikes in population size due to the female-biased progeny (Novitski 1947; Hamilton 1967). 
Y-linked SRDs have been found to occur in nature in the culicine mosquitoes $A e$. aegypti L. and Culex pipiens L., both of which harbour homomorphic sex chromosomes (Gilchrist and Haldane 1974; Fontaine et al. 2017). Males of these two species are heterozygous at the sex-determining locus $(\mathrm{Mm})$ which is located in chromosome 1. The gene determining male sex was recently described in Ae. aegypti (Hall et al. 2015; Turner et al. 2018). The meiotic drive locus, $\mathrm{M}^{\mathrm{D}}$, is closely associated to $\mathrm{M}$, acts in trans, distorting expression at a responder locus that is proximal to and indistinguishable from $\mathrm{m}$, the locus that is homozygous in females. Subtle enhancers and suppressors of $\mathrm{M}^{\mathrm{D}}$ strength have been discovered on all autosomes of Ae. aegypti, as have responder loci of varying sensitivity (Wood and Ouda 1987; Wood and Newton 1991; Cha et al. 2006).

Specific crosses involving the field-caught T37 strain of Ae. aegypti, result in a male-biased population of about 85\% (Shin et al. 2012). Initial cage-population experiments to assess the suitability of the $\mathrm{M}^{\mathrm{D}}$ locus for controlling natural populations of Ae. aegypti, showed females with developed resistance to $\mathrm{M}^{\mathrm{D}}$. Although the mechanism responsible for the resistance remains unknown, the level of distortion ultimately attained was insufficient to achieve effective population control (Hickey and Craig 1966; Robinson 1983).

Since natural resistance to $\mathrm{M}^{\mathrm{D}}$ is common in the field, only native populations that are highly sensitive could be targeted. The efforts involved in the many attempts to apply $\mathrm{M}^{\mathrm{D}}$ for insect control have highlighted the problems that could arise by using naturally occurring distorters for which resistance or rather counteracting alleles are already in existence. Also, their potential to be transferred to other target species is unclear as SRD and responder loci are expected to have co-evolved. Thus, efforts have intensified to develop entirely synthetic SRD strategies, which will be the focus for the remainder of this chapter.

\section{SYNTHETIC SEX RATIO DISTORTERS BASED ON THE X CHROMOSOME SHREDDING MODEL}

Synthetic distorters have the advantage of being unaffected by some, or all of the suppressor alleles that may exist to counteract naturally circulating distorter alleles. This is the case of synthetic SRD systems designed to circumvent the established sex determination pathway, operating independently of it. In the naturally occurring SRDs in Cx. pipiens and Ae. aegypti mosquitoes, cytological observations revealed that during the early stages of male meiosis, the X-equivalent chromosome harbouring the $\mathrm{m}$ locus are fragmented. This is accompanied by an increase in M-bearing gametes and a reduction in the number of females born in the next generation (Newton et al. 1976; Sweeny and Barr 1978).

The observations of $\mathrm{X}$ chromosome fragmentation suggested that a similar system for SRD could be artificially created through endonuclease-mediated cleavage of the $\mathrm{X}$ chromosome during male meiosis. In male heterogametic species, this could be achieved by the expression of a transgene-encoded endonuclease during spermatogenesis that would target and cut the X chromosome at several loci (Fig. 2). Consequently, only gametes with the Y chromosome would be produced or would be functional to achieve fertilization and only males would be generated (Burt 2003). 


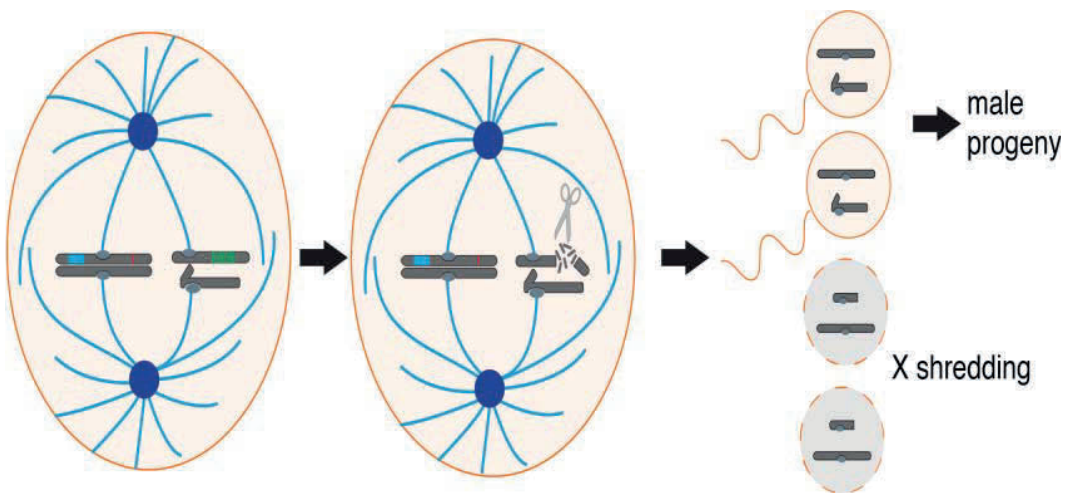

Figure 2. A multicopy target sequence (green bars) on the X chromosome is targeted by an endonuclease (scissors) during spermatogenesis. Shredding of the X chromosome favours the unaffected Y-bearing sperm and results in a male-biased progeny. Blue and red boxes are endonuclease and gRNA genes, respectively.

\subsection{I-PpoI as the First Synthetic Sex Ratio Distorter in the Malaria Mosquito}

A transgenic SRD trait was first developed and tested in the malaria mosquito Anopheles gambiae Giles, expressed from autosomal locations (Windbichler et al. 2007, 2008; Klein et al. 2012). It utilized the Physarum polycephalum Schwein I-PpoI endonuclease, which was driven by the $\beta 2$-tubulin promoter that is specific to spermatogenesis. I-PpoI is an intron-encoded endonuclease mapping in the $28 \mathrm{~S} \mathrm{rDNA}$ locus that selectively cleaves ribosomal DNA sequences.

In An. gambiae, the ribosomal repeats are localised exclusively on the $\mathrm{X}$ chromosome making the targeting and cutting highly specific to the $\mathrm{X}$ chromosome. The expression of wild-type I-PpoI during spermatogenesis resulted in the cleavage of the X chromosome, but also in male sterility. It was found that during fertilisation, mature sperm carrying stable I-PpoI transfers endonuclease protein to the egg, determining the shredding of the maternal $\mathrm{X}$ chromosome and thus sterility.

To reduce the in-vivo half-life of I-PpoI and limit its activity to male meiosis, Galizi and colleagues (Galizi et al. 2014) generated a series of protein variants by sitedirected mutagenesis. They modified amino acid residues involved in the zinc-binding core formation, protein packaging and protein dimerization. Next, they generated transgenic mosquitoes carrying autosomal insertions of each variant of the I-PpoI protein and analysed hatch rate and percentage of SRD in the progeny of hemizygous males crossed to wild-type females. W124A strains, with a mutation in the dimerization domain of I-PpoI, showed the highest hatch rate and SRD in the progeny corresponding to $97.4 \%$ males and a hatch rate close to the control. The SRD phenotype was stably inherited for four consecutive generations by transgenic sons. In five independent laboratory cage experiments with such males it was at least two orders of magnitude more efficient than sterile males. The cage studies also showed that releases of hemizygous distorter males at an overflooding ratio of $3 \mathrm{x}$ to wild-type males, was sufficient to eliminate the An. gambiae cage populations within six generations. 


\subsection{CRISPR-Cas9-Based X Chromosome Shredding to Induce Sex Ratio Distortion}

While the I-PpoI system is a working model for an X chromosome shredder in An. gambiae, it is not transferable to other organisms unless they share the same location of the target rDNA sequences on the X chromosome.

Recent developments in molecular and synthetic biology have provided genome editing tools with great potential to engineer the genome of different species. The most promising is the RNA-guided CRISPR-Cas9 (Clustered Regularly Interspersed Short Palindromic Repeats-CRISPR-associated 9) endonuclease system (Mali et al. 2013). Here, a guide RNA (gRNA) "guides" the endonuclease to the complementary DNA sequence, which is digested by the enzyme generating double-strand breaks. Cas 9 can be used to cleave any complementary target DNA harbouring a PAM (Protospacer Adjacent Motif) sequence, a motif consisting of the three-base-pair, NGG (any nucleobase followed by two guanine nucleobases).

In addition, other RNA-guided endonucleases have recently been discovered, such as $\mathrm{Cpf1}$, smaller than $\mathrm{Cas} 9$ and $\mathrm{A} / \mathrm{T}$ rich genomic-region specific, that may also prove to be as versatile as the Cas9 system (Zetsche et al. 2015). Compared to systems such as zinc-finger nuclease (ZFN), TALE nucleases (TALEN) and homing endonuclease genes (HEGs), RNA-guided systems do not require alteration of the protein to recognize the target sequences. Instead, gRNAs consisting of 18-20 RNA nucleotides are sufficient to lead the endonuclease to its target. Thus, endonucleases with novel specificities can be generated more easily and a larger number of genomic loci can be targeted and cleaved in parallel. Lastly, different gRNAs can be used to target multiple sites simultaneously, thus decreasing the odds of developing resistance alleles.

The CRISPR-Cas9 system, because of its specificity and flexibility, has been tailored to modify the genomes of different organisms, including yeast (DiCarlo et al. 2015), plants (Li et al. 2013), worms (Frokjaer-Jensen 2013), fruit flies (Gratz et al. 2013; Gantz and Bier 2015), the jewel wasp Nasonia vitripennis Walker (Li et al. 2017), mosquitoes (Basu et al. 2015; Gantz et al. 2015; Kistler et al. 2015; Hammond et al. 2016), zebrafish (Hwang et al. 2013), mice (Shen et al. 2013), monkeys (Niu et al. 2014), and human cells (Cong et al. 2013).

Recently, CRISPR-Cas9 was successfully used to act as a SRD system in An. gambiae. As a follow-up of the I-PpoI work, Galizi and colleagues, designed a transformation construct containing the cas 9 gene under the control of the $\beta$-tubulin gene promoter, and a gRNA targeting an X chromosome-linked rDNA sequence distinct from the original I-PpoI target (Galizi et al. 2016). This site was selected because it was conserved among closely related species of the An. gambiae complex, but crucially, was absent from more distantly related insects such as Drosophila. Similarly, to the observations with I-PpoI, the progeny of males from all tested transgenic lines displayed a high SRD with values ranging from $86.1 \%$ to $94.8 \%$ males. The fertility of transgenic males of almost all lines was similar to the wildtype, with hatching rates between $83.6 \%$ and $93.2 \%$. Furthermore, although the $\beta$ tubulin promoter was used to regulate the expression of both Cas9 and I-PpoI (discussed above) endonucleases, the Cas9 protein was not paternally carried over into the fertilised embryo, thus increasing the hatching values and simplifying the generation of SRD. 
Similar to I-PpoI, five consecutive generations of transgenic males stably inherited the SRD phenotype. As was observed for I-PpoI, however, the paternal X chromosome of rare survivor females exhibited both target sequence repeats that were intact indicating that not all of the targets were cleaved, as well as target sequence repeats that were found to be resistant to in-vitro re-cleavage as a possible consequence of DNA miss-repair. This is consistent with CRISPR-Cas9-mediated generation of resistant alleles by non-homologous end joining (NHEJ) or microhomology mediated end joining (MMEJ), repair mechanisms in DNA doublestrand breaks that have been shown to adversely affect CRISPR-based gene drive systems (Hammond et al. 2016). For CRISPR-based SRDs, the likelihood of missrepair leading to overall resistance to $\mathrm{X}$ chromosome shredding and consequently to the failure of population control is greatly reduced as a higher number of $\mathrm{X}$ chromosomal sites are targeted simultaneously.

\subsection{Potential for Establishing CRISPR-Cas9-Based Sex Ratio Distorters in Other Species}

Given the flexibility allowed by CRISPR-based endonucleases, it may now be possible to test whether $\mathrm{X}$ chromosome shredding has the potential to become a universal strategy to genetically control a wide variety of heterogametic insect pests, of both agricultural and public health relevance. However, the proof-of-principle in An. gambiae relies on the peculiar localisation of the mosquito's rDNA genes, which are confined to the $\mathrm{X}$ chromosome, and the availability of an endonuclease that targets these repeats, two characteristics unlikely to be shared by many other insect species.

For X chromosome shredding to become a more widely used approach, what is required is a method to identify motifs repeated exclusively on the $\mathrm{X}$ chromosome, and the ability to engineer endonucleases able to target and cleave these sequences in the male germline.

Recently, a bioinformatic pipeline called Redkmer (for Repeat Extraction and Detection based on kmers, all the possible sub-sequences of length $\mathrm{k}$ from a read obtained through sequencing) used for DNA sequence analysis and comparison, was developed for X chromosome target sequences shredding by CRISPR endonucleases (Papathanos and Windbichler 2018). It relies on long and short read sequencing technology and can identify highly abundant X-specific sequences. Because genome assemblies typically exclude highly repetitive sequences such as satellite DNA, it was designed to not rely on high-quality assembled genomes. Indeed, for many target insect species, high-quality genome assemblies are not available.

The Redkmer pipeline requires as input only whole genome sequencing (WGS) data based on long (e.g. PacBio) and short (e.g. Illumina) unassembled reads of the relevant insect. WGS for the long reads is performed only on male individuals, while short read data are generated from both, male and female individuals, independently. For the selection of highly abundant X chromosome kmers, the pipeline relies on two features: (1) WGS elements mapping on the X chromosome that occur on average twice as often in female compared to male data; (2) overall sequence abundance with $\mathrm{X}$ chromosome specificity. 
Preliminary data in An. gambiae confirmed the potential of the bioinformatic pipeline, revealing X-specific and abundant kmers that overlapped with the known rDNA cluster. The now affordable costs of next-generation sequencing and the increase in computer power, combined with the flexibility of the CRISPR-Cas9 system, will ease the application of SRD to genetically control disease vectors and economically or ecologically damaging invasive species.

\section{CONSIDERATIONS ON THE USE OF SYNTHETIC SEX RATIO DISTORTERS}

SRD systems are subject to two essential limitations. First, SRDs will only work in sexually reproducing species. Second, like the SIT, maintaining SRD traits within a target population requires scheduled releases of new transgenic individuals that need to be continuously reared in considerable numbers rendering the approach costly. As mentioned, this could be eased by linking the SRD trait to the male Y chromosome. Thus, in this case, all male offspring will inherit the transgene that will spread invasively.

For scaling up and potential field testing of the non-driving SRD traits described above, transgene expression should be conditionally repressed. This would enable mass-rearing, as constant backcrossing of a constitutively expressed SRD trait at each generation is unlikely to be a practical method. Conditional expression systems have been successfully used in the field, in combination with dominant lethality, but should be adapted to work in conjunction with CRISPR-based constructs.

\subsection{Difficulty in Turning Non-driving SRD into Driving-SRD Traits}

All synthetic SRD systems described so far are non-driving autosomal distorters. Moving autosomal distorters to the $\mathrm{Y}$ chromosome is, however, far from the straightforward proposition it appeared to be initially. All active distorter transgenes examined by Galizi et al. (unpublished) had the construct inserted on an autosome. Lines with the transgene integrated on either the X or Y chromosome did not show a significant level of I-PpoI expression in the testes. This was explained by the repressive effect of meiotic sex chromosome inactivation (MSCI) that silences unpaired chromatin during meiotic stages of spermatogenesis. Inactivation of transgenes integrated on the $\mathrm{X}$ chromosome has been described before in mosquitoes (Magnusson et al. 2012; Papa et al. 2017). MSCI may turn out to be a significant obstacle in turning non-driving SRD into driving-SRD traits, as its biological function may be that of policing SD traits.

\subsection{Advantages of the Standard Autosomal SRD Systems}

One advantage of the standard autosomal SRD systems based on RNA-guided endonucleases is that they can be engineered to work, at least in principle, in most heterogametic species and therefore suppress the population of many target organisms. 
Recently the precise deletion of chromosomes in cell lines of mammals (Adikusuma et al. 2017) has been demonstrated and indicates that this system could also work in higher organisms. From an ecological standpoint, a second advantage is the reduced risk of invasion compared to driving Y-linked SRDs or compared to other gene drive elements that are being developed for the purpose of population replacement (Gantz and Bier 2016; Hammond et al. 2016). Once released, the frequency of the SD alleles decreases instead of increasing in the population, a desirable feature to avoid permanent ecosystems alterations (Oye et al. 2014).

\subsection{Engineering Multiple gRNAs to Reduce Generation of Resistance Alleles}

In RNA-guided SRD systems there is the need to understand the likelihood at which resistant alleles will develop in the target sequences (Bull 2015). The tendency of natural selection to favour equal sex ratios exerts pressure on the NHEJ (nonhomologous end joining) repair system that may generate resistance alleles via insertion and deletions in target sequences. In addition, natural sequence polymorphism between individuals of the same population could also prevent cutting. Therefore, to reduce the growth of resistance alleles, it is critical to engineer different gRNAs cutting simultaneously, including some that target conserved regions of essential genes. The degree to which $\mathrm{X}$ chromosome shredding systems can rely on the existence of numerous repetitive sequences of the same target on the $\mathrm{X}$ chromosome, may directly determine the likelihood of the rise of resistance alleles, although further research is required in this area.

\subsection{Environmental, Ecological, and Regulatory Challenges}

The release of transgenically modified organisms for population control is challenged by a series of environmental, ecological, and regulatory difficulties. Transgenic males should be able to mate and scout for females to the same extent as wild-type males (Lacroix et al. 2012). A different behaviour would drop the chances of spreading to remote regions reducing the success of population control. Different factors can decrease the competitiveness of males such as mass-rearing, inbreeding, transgene expression and its insertion site in the genome (Catteruccia et al. 2003; Reed et al. 2003; Baeshen et al. 2014). The use of the PhiC31 integration system that provides precisely mapped docking sites, has helped to reduce the position effect on gene expression (Amenya et al. 2010).

Genetic variation of wild-type individuals is another feature that can influence the success of the release operation. Polymorphisms in the gRNA target site can compromise the ability of the endonuclease to cleave the DNA. Different strains can have slightly dissimilar mating behaviours or live in distinctive ecological niches making their control even more difficult. These are all features that should be considered when applying genetic engineering to control vector-borne disease or agricultural pests. 
Ultimately, the release of transgenically modified individuals causes environmental and safety challenges that should be addressed in each individual case. Ecological and molecular containment strategies should be considered when designing RNA-guided SRD systems. The chances of the SRD trait spreading to nontargeted species, and horizontal transfer of the transgenes must be safely reduced before releasing the modified individuals (David et al. 2013; Nielsen, this volume). Species-specific targeting sequences and promoters to regulate the endonuclease expression should also prevent lateral gene transfer beyond target populations (Oye et al. 2014).

Confined laboratories, with high containment levels and only small-field tests, should initially be used to determine the safety and specificity of the transgene modification. An open discussion with regulatory agencies, the scientific community and the public is fundamental to inform on the risks and benefits of using geneticengineering technologies to control vector-borne diseases and alleviate the economic burden inflicted by agricultural pests (Oye et al. 2014).

\section{OTHER APPROACHES TO ACHIEVE SYNTHETIC SEX RATIO DISTORTION}

We have focussed our discussion on $\mathrm{X}$ chromosome shredding as a paradigm for developing synthetic SRDs. However, other approaches are also conceivable to achieve the same goal. Recently, the long-elusive male-determining genes of a number of important pest and vector species have been identified. These include the $\mathrm{Y}$ chromosome-linked male-determining genes in mosquitoes An. gambiae and An. stephensi Liston, the M-locus linked gene in Ae. aegypti, the mobile splicing factor in M. domestica L. and the MoY factor in Ceratitis capitata (Wiedemann) (Hall et al. 2015; Criscione et al. 2016; Krzywinska et al. 2016; Sharma et al. 2017; Meccariello et al. 2019).

Knowing the sex-determining genes in each of these species will allow designing synthetic SRDs. In addition, the use of a nuclease-based gene drive could ensure the transmission of the synthetic SRDs to the entire progeny and bring more rapidly the population to collapse (Kyrou et al. 2018; Häcker and Schetelig, this volume).

Finally, caution should be used when sex-determining genes play functional roles in other essential pathways, such as in dosage compensation. Their multiple functions may interfere with some strategies to manipulate sex ratios, for example through the lethality of transgenic male-determination signals on chromosomes other than the $\mathrm{Y}$ chromosome.

\section{REFERENCES}

Adikusuma, F., N. Williams, F. Grutzner, J. Hughes, and P. Thomas. 2017. Targeted deletion of an entire chromosome using CRISPR/Cas9. Molecular Therapy 25: 1736-1738.

Amenya, D. A., M. Bonizzoni, A. T. Isaacs, N. Jasinskiene, H. Chen, O. Marinotti, G. Yan, and A. A. James. 2010. Comparative fitness assessment of Anopheles stephensi transgenic lines receptive to site-specific integration. Insect Molecular Biology 19: 263-269.

Argasinski, K. 2013. The dynamics of sex ratio evolution: From the gene perspective to multilevel selection. PLoS One 8: e60405. 
Baeshen, R., N. E. Ekechukwu, M. Toure, D. Paton, M. Coulibaly, S. F. Traore, and F. Tripet. 2014. Differential effects of inbreeding and selection on male reproductive phenotype associated with the colonization and laboratory maintenance of Anopheles gambiae. Malaria Journal 13: 19.

Basu, S., A. Aryan, J. M. Overcash, G. H. Samuel, M. A. Anderson, T. J. Dahlem, K. M. Myles, and Z. N. Adelman. 2015. Silencing of end-joining repair for efficient site-specific gene insertion after TALEN/CRISPR mutagenesis in Aedes aegypti. Proceedings of the National Academy of Sciences of the USA 112: 4038-4043.

Bull, J. J. 2015. Evolutionary decay and the prospects for long-term disease intervention using engineered insect vectors. Evolution Medicine and Public Health 2015: 152-166.

Burt, A. 2003. Site-specific selfish genes as tools for the control and genetic engineering of natural populations. Proceedings of the Royal Society B: Biological Sciences 270: 921-928.

Catteruccia, F., H. C. Godfray, and A. Crisanti. 2003. Impact of genetic manipulation on the fitness of Anopheles stephensi mosquitoes. Science 299: 1225-1227.

Cha, S. J., D. D. Chadee, and D. W. Severson. 2006. Population dynamics of an endogenous meiotic drive system in Aedes aegypti in Trinidad. American Journal of Tropical Medicine and Hygiene 75: $70-77$.

Cong, L., F. A. Ran, D. Cox, S. Lin, R. Barretto, N. Habib, P. D. Hsu, X. Wu, W. Jiang, L. A. Marraffini, and F. Zhang. 2013. Multiplex genome engineering using CRISPR/Cas systems. Science 339: 819-823.

Craig, G. B., W. A. Hickey, and R. C. VandeHey. 1960. An inherited male-producing factor in Aedes aegypti. Science 132: 1887-1889.

Criscione, F., Y. Qi, and Z. Tu. 2016. GUY1 confers complete female lethality and is a strong candidate for a male-determining factor in Anopheles stephensi. eLife 5: e19281.

David, A. S., J. M. Kaser, A. C. Morey, A. M. Roth, and D. A. Andow. 2013. Release of genetically engineered insects: A framework to identify potential ecological effects. Ecology and Evolution 3: 4000-4015.

DiCarlo, J. E., A. Chavez, S. L. Dietz, K. M. Esvelt, and G. M. Church. 2015. Safeguarding CRISPRCas9 gene drives in yeast. Nature Biotechnology 33: 1250-1255.

Dyck, V. A., J. Hendrichs, and A. S. Robinson (eds.). 2021. Sterile Insect Technique - Principles and practice in Area-Wide Integrated Pest Management. Second Edition. CRC Press, Boca Raton, Florida, USA. 1200 pp.

Fisher, R. A. 1958. The genetical theory of natural selection. Dover, New York, NY, USA. 291 pp.

Fontaine, A., I. Filipovic, T. Fansiri, A. A. Hoffmann, C. Cheng, M. Kirkpatrick, G. Rasic, and L. Lambrechts. 2017. Extensive genetic differentiation between homomorphic sex chromosomes in the mosquito vector, Aedes aegypti. Genome Biology and Evolution 9: 2322-2335.

Frokjaer-Jensen, C. 2013. Exciting prospects for precise engineering of Caenorhabditis elegans genomes with CRISPR/Cas9. Genetics 195: 635-642.

Galizi, R., L. A. Doyle, M. Menichelli, F. Bernardini, A. Deredec, A. Burt, B. L. Stoddard, N. Windbichler, and A. Crisanti. 2014. A synthetic sex ratio distortion system for the control of the human malaria mosquito. Nature Communications 5: 3977.

Galizi, R., A. Hammond, K. Kyrou, C. Taxiarchi, F. Bernardini, S. M. O'Loughlin, P. A. Papathanos, T. Nolan, N. Windbichler, and A. Crisanti. 2016. A CRISPR-Cas9 sex-ratio distortion system for genetic control. Scientific Reports Nature 6: 31139.

Gantz, V. M., and E. Bier. 2015. Genome editing. The mutagenic chain reaction: A method for converting heterozygous to homozygous mutations. Science 348: 442-444.

Gantz, V. M., and E. Bier. 2016. The dawn of active genetics. Bioessays 38: 50-63.

Gantz, V. M., N. Jasinskiene, O. Tatarenkova, A. Fazekas, V. M. Macias, E. Bier, and A. A. James. 2015. Highly efficient Cas9-mediated gene drive for population modification of the malaria vector mosquito Anopheles stephensi. Proceedings of the National Academy of Sciences of the USA 112: E6736-6743.

Gilchrist, B. M., and J. B. S. Haldane. 1974. Sex linkage and sex determination in a mosquito Culex molestus. Hereditas 33: 175-190.

Gratz, S. J., A. Cummings, J. N. Nguyen, D. C. Hamm, L. K. Donohue, M. M. Harrison, J. Wildonger, and K. M. O'Connor-Giles. 2013. Genome engineering of Drosophila with the CRISPR RNA-guided Cas9 nuclease. Genetics 194: 1029-1035. 
Hall, A. B., S. Basu, X. Jiang, Y. Qi, V. A. Timoshevskiy, J. K. Biedler, M. V. Sharakhova, R. Elahi, M. A. Anderson, X. G. Chen, I. V. Sharakhov, Z. N. Adelman, and Z. Tu. 2015. SEX DETERMINATION. A male-determining factor in the mosquito Aedes aegypti. Science 348: 12681270 .

Hamilton, W. D. 1967. Extraordinary sex ratios. A sex-ratio theory for sex linkage and inbreeding has new implications in cytogenetics and entomology. Science 156: 477-488.

Hammer, M. F. 1991. Molecular and chromosomal studies on the origin of thaplotypes in mice. The American Naturalist 137: 359-365.

Hammond, A., R. Galizi, K. Kyrou, A. Simoni, C. Siniscalchi, D. Katsanos, M. Gribble, D. Baker, E. Marois, S. Russell, A. Burt, N. Windbichler, A. Crisanti, and T. Nolan. 2016. A CRISPR-Cas9 gene drive system targeting female reproduction in the malaria mosquito vector Anopheles gambiae. Nature Biotechnology 34: 78-83.

Hickey, W. A., and G. B. Craig. 1966. Genetic distortion of sex ratio in a mosquito, Aedes aegypti. Genetics 53: 1177-1196.

Hwang, W. Y., Y. Fu, D. Reyon, M. L. Maeder, S. Q. Tsai, J. D. Sander, R. T. Peterson, J. R. Yeh, and J. K. Joung. 2013. Efficient genome editing in zebrafish using a CRISPR-Cas system. Nature Biotechnology 31: 227-229.

Kistler, K. E., L. B. Vosshall, and B. J. Matthews. 2015. Genome engineering with CRISPR-Cas9 in the mosquito Aedes aegypti. Cell Reports 11: 51-60.

Klein, T. A., N. Windbichler, A. Deredec, A. Burt, and M. Q. Benedict. 2012. Infertility resulting from transgenic I-PpoI male Anopheles gambiae in large cage trials. Pathogens and Global Health 106: 2031 .

Krzywinska, E., N. J. Dennison, G. J. Lycett, and J. Krzywinski. 2016. A maleness gene in the malaria mosquito Anopheles gambiae. Science 353: 67-69.

Kyrou, K., A. M. Hammond, R. Galizi, N. Kranjc, A. Burt, A. K. Beaghton, T. Nolan, and A. Crisanti. 2018. A CRISPR-Cas9 gene drive targeting doublesex causes complete population suppression in caged Anopheles gambiae mosquitoes. Nature Biotechnology 36: 1062-1066.

Lacroix, R., A. R. McKemey, N. Raduan, L. Kwee Wee, W. Hong Ming, T. Guat Ney, A. A. S. Rahidah, S. Salman, S. Subramaniam, O. Nordin, A. T. N. Hanum, C. Angamuthu, S. Marlina Mansor, R. S. Lees, N. Naish, S. Scaife, P. Gray, G. Labbé, C. Beech, D. Nimmo, L. Alphey, S. S. Vasan, L. Han Lim, A. N. Wasi, and S. Murad. 2012. Open field release of genetically engineered sterile male Aedes aegypti in Malaysia. PLoS One 7(8): e42771.

Li, J. F., J. E. Norville, J. Aach, M. McCormack, D. Zhang, J. Bush, G. M. Church, and J. Sheen. 2013. Multiplex and homologous recombination-mediated genome editing in Arabidopsis and Nicotiana benthamiana using guide RNA and Cas9. Nature Biotechnology 31: 688-691.

Li, M., L. Y. C. Au, D. Douglah, A. Chong, B. J. White, P. M. Ferree, and O. S. Akbari. 2017. Generation of heritable germline mutations in the jewel wasp Nasonia vitripennis using CRISPR/Cas9. Scientific Reports Nature 7: 901.

Lyttle, T. W. 1991. Segregation distorters. Annual Review of Genetics 25: 511-557.

Magnusson, K., G. J. Lycett, A. M. Mendes, A. Lynd, P. A. Papathanos, A. Crisanti, and N. Windbichler. 2012. Demasculinization of the Anopheles gambiae X chromosome. BMC Evolutionary Biology 12: 69.

Mali, P., K. M. Esvelt, and G. M. Church. 2013. Cas9 as a versatile tool for engineering biology. Nature Methods 10: 957-963.

Meccariello, A., M. Salvemini, P. Primo, B. Hall, P. Koskinioti, M. Dalíková, A. Gravina, M. A. Gucciardino, F. Forlenza, M. E. Gregoriou, D. Ippolito, S. M. Monti, V. Petrella, M. M. Perrotta, S. Schmeing, A. Ruggiero, F. Scolari, E. Giordano, K. T. Tsoumani, F. Marec, N. Windbichler, J. Nagaraju, K. P. Arunkumar, K. Bourtzis, K. D. Mathiopoulos, J. Ragoussis, L. Vitagliano, Z. Tu, P. A. Papathanos, M. D. Robinson, and G. Saccone. 2019. Maleness-on-the-Y (MoY) orchestrates male sex determination in major agricultural fruit fly pests. Science 365 (6460): 14571460:

Mercot, H., B. Llorente, M. Jacques, A. Atlan, and C. Montchamp-Moreau. 1995. Variability within the Seychelles cytoplasmic incompatibility system in Drosophila simulans. Genetics 141: 1015-1023.

Newton, M. E., R. J. Wood, and D. I. Southern. 1976. A cytogenetic analysis of meiotic drive in the mosquito Aedes aegypti. Genetica 46: 297-318. 
Niu, Y, B. Shen, Y. Cui, Y. Chen, J. Wang, L. Wang, Y. Kang, X. Zhao, W. Si, W. Li, A. P. Xiang, J. Zhou, X. Guo, Y. Bi, C. Si, B. Hu, G. Dong, H. Wang, Z. Zhou, T. Li, T. Tan, X. Pu, F. Wang, S. Ji, Q. Zhou, X. Huang, W. Ji, and J. Sha. 2014. Generation of gene-modified cynomolgus monkey via Cas9/RNA-mediated gene targeting in one-cell embryos. Cell 156: 836-843.

Novitski, E. 1947. Genetic analysis of anomalous sex ratio condition in Drosophila affinis. Genetics 32 : $526-534$.

Oye, K. A., K. Esvelt, E. Appleton, F. Catteruccia, G. Church, T. Kuiken, S. B. Lightfoot, J. McNamara, A. Smidler, and J. P. Collins. 2014. Biotechnology. Regulating gene drives. Science 345: 626-628.

Papa, F., N. Windbichler, R. M. Waterhouse, A. Cagnetti, R. D'Amato, T. Persampieri, M. K. N. Lawniczak, T. Nolan, and P. A. Papathanos. 2017. Rapid evolution of female-biased genes among four species of Anopheles malaria mosquitoes. Genome Research 27: 1536-1548.

Papathanos, P. A., and N. Windbichler. 2018. Redkmer: An assembly-free pipeline for the identification of abundant and specific X-chromosome target sequences for X-shredding by CRISPR endonucleases. CRISPR Journal 1: 88-98.

Reed, D. H., E. H. Lowe, D. A. Briscoe, and R. Frankham. 2003. Fitness and adaptation in a novel environment: Effect of inbreeding, prior environment, and lineage. Evolution 57: 1822-1828.

Robinson, A. S. 1983. Sex-ratio manipulation in relation to insect pest control. Annual Review Genetics 17: 191-214.

Schliekelman, P., S. Ellner, and F. Gould. 2005. Pest control by genetic manipulation of sex ratio. Journal of Economic Entomology 98: 18-34.

Sharma, A., S. D. Heinze, Y. Wu, T. Kohlbrenner, I. Morilla, C. Brunner, E. A. Wimmer, L. van de Zande, M. D. Robinson, L. W. Beukeboom, and D. Bopp. 2017. Male sex in houseflies is determined by $M d m d$, a paralog of the generic splice factor gene $C W C 22$. Science 356: 642-645.

Shen, B., J. Zhang, H. Wu, J. Wang, K. Ma, Z. Li, X. Zhang, P. Zhang, and X. Huang. 2013. Generation of gene-modified mice via Cas9/RNA-mediated gene targeting. Cell Research 23: 720723.

Shin, D., A. Mori, and D. W. Severson. 2012. Genetic mapping a meiotic driver that causes sex ratio distortion in the mosquito Aedes aegypti. Journal of Heredity 103: 303-307.

Sweeny, T. L., and A. R. Barr. 1978. Sex ratio distortion caused by meiotic drive in a mosquito, Culex pipiens L. Genetics 88: 427-446.

Thomas, D. T., C. A. Donnelly, R. J. Wood, and L. S. Alphey. 2000. Insect population control using a dominant, repressible, lethal genetic system. Science 287: 2474-2476.

Turner, J., R. Krishna, A. E. van't Hof, E. R. Sutton, K. Matzen, and A. C. Darby. 2018. The sequence of a male-specific genome region containing the sex determination switch in Aedes aegypti. Parasites and Vectors 11: 549.

Windbichler, N., P. A. Papathanos, F. Catteruccia, H. Ranson, A. Burt, and A. Crisanti. 2007. Homing endonuclease mediated gene targeting in Anopheles gambiae cells and embryos. Nucleic Acids Research 35: 5922-5933.

Windbichler, N., P. A. Papathanos, and A. Crisanti. 2008. Targeting the $X$ chromosome during spermatogenesis induces $\mathrm{Y}$ chromosome transmission ratio distortion and early dominant embryo lethality in Anopheles gambiae. PLoS Genetics 4: e1000291.

Wood, R. J., and N. A. Ouda. 1987. The genetic basis of resistance and sensitivity to the meiotic drive gene D in the mosquito Aedes aegypti L. Genetica 72: 69-79.

Wood, R. J., and M. E. Newton. 1991. Sex-ratio distortion caused by meiotic drive in mosquitoes. The American Naturalist 137: 379-391.

Zetsche, B, J. S. Gootenberg, O. O. Abudayyeh, I. M. Slaymaker, K. S. Makarova, P. Essletzbichler, S. E. Volz, J. Joung, J. van der Oost, A. Regev. E. V. Koonin, and F. Zhang. 2015. Cpf1 is a single RNA-guided endonuclease of a class 2 CRISPR-Cas system. Cell 163: 759-771.

Zimmering, S, L. Sandler, and B. Nicoletti. 1970. Mechanisms of meiotic drive. Annual Review Genetics 4: 409-436. 